\title{
Gestión del conocimiento en PYMES del sector servicios: Una aproximación empírica en el Estado de México
}

\author{
Volumen XX No (1). Enero-Junio 2020. Pág. 11-24
}

ISSN: 0121-1048 IMPRESO ISSN: 2422-3220 EN LÍNEA

\section{Caldera-González, Diana del Consuelo.}

División de Ciencias Económico Administrativas, Universidad de

Guanajuato, Fraccionamiento 1 Col. El Establo, CP. 36250,

Guanajuato, México. dccaldera@ugto.mx *autor corresponsal

\section{Escamilla-Salazar, Zugaide}

Universidad Autónoma del Estado de México, Centro

Universitario Atlacomulco,

Estado de México, México.

zescamillas@uaemex.mx

\section{Ruiz-Lara, Fortino}

Universidad Autónoma del Estado de México, Centro

Universitario Atlacomulco,

Estado de México, México.

fortinoruiz93@gmail.com

\section{Resumen}

El impacto que tienen las pequeñas y medianas empresas (Pymes) en el desarrollo de las sociedades es innegable, a pesar de los retos que enfrentan ante un mundo incierto y cambiante. Este ambiente las obliga a ser más competitivas y una manera de lograrlo es planificando y controlando el qué hacer y el saber hacer en la organización como parte de la integración de la gestión del conocimiento. El objetivo de este trabajo es identificar los indicadores más significativos del factor gestión del conocimiento en Pymes del sector servicios en un municipio de la Zona Norte del Estado de México. Para tal efecto se utiliza la técnica estadística de tabla de contingencia. Los principales resultados muestran que el indicador imitación es significativo para el grupo de empresarios mujeres y hombres de la muestra de esta investigación, lo cual alerta acerca de la falta de planeación para el tema de gestión de conocimiento en este tipo de empresas.

Palabras clave: Gestión de conocimiento, Factores, Competitividad, Pymes, México.

Códigos JEL: L1, L2, D8.

\footnotetext{
• Citar: Caldera-González, Diana del Consuelo, Escamilla-Salazar, Zugaide y Ruiz-Lara, Fortino (2020). Gestión del Conocimiento en
} PYMES del Sector Servicios: Una aproximación empírica en el Estado de México. Inquietud Empresarial, 20(1), 11-24. 


\title{
Management of knowledge in SME's on services sector. An empirical approach in Mexico's state
}

\begin{abstract}
The impact that small and medium-sized enterprises (SMEs) have on the development of societies is undeniable, despite the challenges they face in an uncertain and changing world. This environment forces them to be more competitive and one way to achieve this is by planning and controlling what to do and know-how in the organization as part of the integration of knowledge management. The objective of this work is to identify the most significant indicators of the knowledge management factor in SMEs in the service sector in a municipality in the Northern Zone of the State of Mexico. For this purpose, the statistical contingency table technique is used. The main results show that the imitation indicator is significant for the group of female and male entrepreneurs in the sample of this research, which alerts us to the lack of planning for the issue of knowledge management in this kind of companies.
\end{abstract}

Key words: Knowledge management, Factors, Competitiveness, SME's, Mexico.

JEL Classification: L1, L2, D8.

\section{INTRODUCCIÓN}

En México, la sociedad vive donde el ritmo va en función del conocimiento. Por lo tanto, la educación ha requerido una transformación basada en una nueva visión. Esta debe permitir una formación integral a los estudiantes, también debe considerar las competencias necesarias para aprender a lo largo de la vida (conocer, hacer y convivir). Todas ellas, imprescindibles para afrontar los retos y oportunidades que sustentan el desarrollo del país (Hernández, 2010).

En los últimos años las pequeñas $\mathrm{y}$ medianas empresas (Pymes) se han convertido en una pieza fundamental de las economías mundiales, considerándolas el motor principal para el crecimiento y progreso de los países (ONU, 2018). En este contexto, se manifiesta que este tipo de negocios representa más del 90 por ciento del total de las empresas generando el 50 por ciento del producto interno bruto (PIB) a nivel mundial (BANSEFI, 2018).

En este sentido, las Pymes en un entorno globalizado son un factor determinante para la economía, a pesar de las dificultades en cuanto al acceso de financiamiento y el alto índice de fracaso con el que se enfrentan. Por consiguiente, el mercado global obliga a las empresas a generar mayores fortalezas mediante ventajas competitivas sostenibles, lo cual ha llevado a que estas empresas se vean en la necesidad de innovar y adaptarse a cambios tales como la gestión del conocimiento y el uso de las tecnologías de información (Marulanda, Giraldo \& López, 2012).

Por tal motivo y por causa de la complejidad que generan los diferentes cambios a los que se enfrentan este tipo de organizaciones, es indispensable la 
adaptación a un ritmo de mayor exigencia para el cual la única manera de ser competitivas radica en planificar y controlar el qué hacer y el saber hacer, esto como parte fundamental de la gestión del conocimiento, concepto que es integrado a las necesidades generales de las Mipymes (Mirabal, 2015). En efecto, la gestión del conocimiento se vuelve una poderosa herramienta para el éxito de estas empresas, logrando trascender mucho más que cualquier otro elemento organizacional dentro de ellas generando valor a largo plazo (Arriaga \& Gómez, 2014).

En la década de los noventa surge el concepto de gestión de conocimiento como herramienta tecnológica de la información. Con la evolución se ha enfocado al aspecto humano resaltando el conocimiento en experiencias, habilidades, actitudes $y$ aptitudes, por lo que no es un contexto definido y lineal dentro de las empresas, sino que se adecúa en función de las necesidades de la organización que lo implementa (Vargas \& Moreno, 2005).

Existen diversos enfoques o técnicas los cuales han sido utilizados con el afán de generar una mayor rentabilidad económica en las empresas, mostrando una situación de controversia y de teorías opuestas, ya que cada enfoque señala ser la solución para los problemas en las empresas (Wilson, 2002). Tal es el caso de Cabrera et al. (2015) quienes argumentan que los sistemas de gestión basados en calidad en conjunto con las normas internacionales (ISO por sus siglas en inglés) facilitan el desempeño de las funciones internas dentro de la organización y además de esto generan una imagen corporativa positiva ante la sociedad, por lo que entra en desacuerdo con el enfoque de Bustelo y Amarilla (2001) quienes afirman que la teoría de gestión de conocimiento es capaz de responder y adaptarse a las últimas innovaciones en materia tecnológica, en el procesamiento de la información, por mencionar dos enfoques opuestos.

En México, la gestión del conocimiento no ha sido suficientemente estudiada de manera empírica, tal y como lo señalan Maldonado, Martínez y García (2012), pocas las empresas que conocen e implementan la gestión de conocimiento.

Así, el objetivo general de este trabajo es identificar los indicadores más significativos del factor transferencia de gestión del conocimiento en Pymes del sector servicios en un municipio de la zona norte del estado de México. Para cumplir lo anterior, este trabajo se encuentra estructurado en cuatro apartados. En el primero, se presenta una reflexión teórica la cual permite comprender los términos de gestión del conocimiento y transferencia de conocimiento desde un enfoque organizacional; el segundo apartado, se particulariza la metodología describiendo el tipo de investigación a desarrollar, las técnicas de recolección de datos, el objeto de estudio, así como la determinación de la población y definición de la muestra; por lo que respecta en el tercer apartado, se discuten los resultados obtenidos de la investigación; en el último apartado, se detalla las conclusiones en donde da pauta a exponer los hallazgos y convicciones encontradas en el estudio.

\section{MARCo TEÓRICO}

\subsection{Gestión del conocimiento}

En el ámbito empresarial, la competitividad y la incertidumbre obligan a transformar la realidad empresarial, en donde la capacidad de innovación forma parte de la adaptación al entorno, por ende, la gestión del 
conocimiento y la capacidad de innovación se convierten elementos clave para el éxito (Barrios, Olivero \& Acosta, 2017). En este tenor, la revisión de la literatura muestra diversas apreciaciones sobre el constructo gestión del conocimiento, tal es el caso de Davenport y Prusak, (2001, citado en Nagles, 2007) quienes lo definen como:

Un proceso lógico, organizado y sistemático para producir, transferir y aplicar en situaciones concretas una combinación armónica de saberes, experiencias, valores, información contextual y apreciaciones expertas que proporcionan un marco para su evaluación e incorporación de nuevas experiencias e información. (p. 77)

Por su parte, Vargas y Moreno (2005) hacen énfasis en la nueva economía del conocimiento, donde éste es capaz de reemplazar al capital como un recurso crítico; así, la capacidad de generar, transformar y transmitir el conocimiento se convierte en la principal ventaja competitiva de las organizaciones.

Por ende, la gestión de conocimiento se ha reconocido como el componente clave en los procesos de negocio, ya que permite generar $\mathrm{y}$ fortalecer una ventaja competitiva sostenible como parte de los paradigmas de la dirección estratégica, con el propósito de responder, adaptar y sostenerse ante los cambios del entorno mediante el conocimiento, innovación, uso de las tecnologías de información y mejoramiento continuo (Marulanda, López \& Mejía, 2013).

Es preciso señalar que, aunque los conceptos de información y conocimiento se utilizan con frecuencia como sinónimos, son distintos; la información consiste en flujos de mensajes, y el conocimiento es creado cuando ese flujo de información encuentra significado en el sujeto (Montreal, 2000). De forma semejante, Valencia (2010) define al conocimiento como la información personalizada que el individuo posee, la cual se relaciona con su aprendizaje formal e informal a lo largo de su vida.

Existen dos tipos básicos de conocimiento: el conocimiento explícito, que se puede expresar en un lenguaje formal y sistemático $\mathrm{y}$, por tanto, es posible procesarlo, transmitirlo y almacenarlo; y el conocimiento tácito, el cual cuenta con elementos cognoscitivos, paradigmas, creencias o perspectivas y otros elementos como la experiencia empírica, las habilidades $\mathrm{y}$ competencias que adquiere el individuo de manera natural (Montreal, 2000; Rodríguez, 2006; Nagles, 2007).

De esta manera, ambos conocimientos deben ser articulados para extraer la información de los sujetos que forman parte de un organismo, es aquí donde la gestión de conocimiento juega un papel significativo en la conducta empresarial ya que da pauta a la resistencia mental es decir a mentes arraigadas que influye sobre nuestro modo de comprender y actuar.

Para entender cómo se debe emplear la gestión del conocimiento en las organizaciones, se muestra en la Tabla 1, algunos enfoques sobre la perspectiva y los factores que se relaciona con la gestión del conocimiento. (Ver Tabla 1)

El proceso que guía la gestión del conocimiento es complejo, por lo cual existen diferentes perspectivas desde el punto de vista estratégico donde se hace hincapié en el diseño de la estructura organizacional, así como en el enfoque que da pauta a una ventaja sostenible como parte del paradigma de la dirección estratégica y profundiza en la cultura organizacional, en contraste con la 
gestión del conocimiento como fuente de innovación, la cual particulariza la creación y adaptación, lo que da como resultado la ventaja competitiva sostenible; de la misma manera se tiene la gestión del conocimiento vista desde el enfoque operativo centrándose en el uso de las tecnologías de información.

Dicho de otro modo, el conocimiento tiene que ver con la creatividad y la capacidad de innovación de los individuos que integran la organización y sus interacciones, sin dejar a un lado el término de transferencia de conocimiento que se puede considerar parte del proceso de gestión del conocimiento o bien como la herramienta que permite la interconexión de las aportaciones de individuo y la sinergia entre todas ellas.

TABLA 1. ENFOQUES DE LA GESTIÓN DEL CONOCIMIENTO

\begin{tabular}{|c|c|c|}
\hline Perspectiva/enfoque & Postura & Factores \\
\hline Gestión estratégica del conocimiento & Diseño de estructuras organizativas & $\begin{array}{l}\text {-Conocimiento } \\
\text {-Estrategia empresarial } \\
\text {-Desarrollo de profesionales de } \\
\text { conocimiento }\end{array}$ \\
\hline Gestión operativa del conocimiento & $\begin{array}{l}\text { Usos de las Tecnologías de Información de } \\
\text { Comunicación }\end{array}$ & $\begin{array}{l}\text {-Información } \\
\text {-Organización } \\
\text {-Distribución } \\
\text {-Empleados }\end{array}$ \\
\hline $\begin{array}{l}\text { Gestión del conocimiento como } \\
\text { fuente de innovación }\end{array}$ & $\begin{array}{l}\text { Maximizar el valor de una organización mediante la } \\
\text { creación y adaptación de valor }\end{array}$ & $\begin{array}{l}\text {-Necesidad } \\
\text {-Conocimiento } \\
\text {-Experiencias } \\
\text {-Habilidades } \\
\text {-Actitudes } \\
\text {-Aptitudes }\end{array}$ \\
\hline $\begin{array}{l}\text { Gestión del conocimiento como } \\
\text { ventaja sostenible }\end{array}$ & Paradigma en dirección estratégica & $\begin{array}{l}\text {-Creación del valor } \\
\text {-Cultura organizacional } \\
\text {-Motivación } \\
\text {-Actitudes }\end{array}$ \\
\hline
\end{tabular}

Fuente: elaboración propia con base en Ordoñez (2001); Vargas \& Moreno (2005); Nagles (2007).

La transferencia de conocimiento está condicionada por factores organizativos tales como: liderazgo, cultura, clima, tecnología y estructura (Vargas \& Moreno, 2005), es decir, la generación de organizaciones que aprenden. Como lo mencionan López, Alvarado, Fong y González (2019), la ventaja competitiva de las empresas depende de sus recursos y capacidades, así como de la manera en que éstos se utilizan, lo cual indudablemente se relaciona con el tema de la gestión del conocimiento.

La gestión de conocimiento no es lineal. Autores como Ruiz y García (2013) lo definen como aquella que refiere a la identificación y el aprovechamiento del conocimiento colectivo en una organización para ayudarla a competir. Por otro lado, Bustelo y Amarilla (2001) lo definen como un mecanismo para el logro efectivo de los objetivos a través de actividades encaminadas a crear, usar y compartir conocimientos en una organización. Por su parte, Mirabal (2015) en un estudio más reciente lo asocian con la capacidad de generar o potenciar competencias para ser más eficientes.

Así, la revisión de la literatura permitió detectar trabajos provenientes de países diversos, tal es el caso de Bustelo, y Amarilla (2001), Cabrera y Rincón (2001), Davidson y 
Voss (2002), Dalkir (2005), Črnjar (2006), Rodríguez (2006), Pérez (2009), Rodríguez, Pedraja, Delgado y Ponce (2010), Bernal, Fracica y Frost (2012), Bagnoli y Vedovato (2012), Maldonado, Martínez y García (2012), Marulanda, Giraldo y López (2012), Ruiz y García (2013), y similares al nuestro está el de Jingjing (2007), Byung, Hyunsoo, Tae (2018), Yonghai, Aiqun, Houlei, Shugang (2009), y el de Martínez (2013).

Cabe destacar que el corte de investigación de los trabajos referidos en el párrafo anterior es de tipo teórico, así, el porcentaje de los trabajos con corte empírico es menor, así mismo es preciso mencionar que los trabajos tanto teóricos como empíricos en el tema de gestión del conocimiento en Pymes del sector servicios en México son escasos. En la revisión teórica realizada, se encontró un solo caso aplicado al sector servicios en las Pymes del estado de Chihuahua, México, realizado por Gutiérrez et al. (2015), y un solo trabajo enfocado al sector manufacturero en las Pymes del estado de Aguascalientes, México, realizado por Cuevas, Rangel y Hernández (2014). En ambos estudios se muestra que la gestión del conocimiento es fundamental en este contexto tan competitivo.

Ciertamente existen escasas contribuciones acerca de la gestión del conocimiento en México en Pymes, a pesar de que este tipo de organizaciones representan el 95 por ciento del total de las empresas en el país, las cuales aportan el 52 por ciento del Producto Interno Bruto a nivel nacional de acuerdo con datos extraídos del Instituto Nacional de Geografía y Estadística (INEGI, 2016).

Para la presente investigación se ha tomado como base teórica el modelo propuesto por Martínez (2013), quien integra dos grupos de factores para el estudio de la gestión del conocimiento. Primeramente, el de transferencia de conocimiento que a su vez integran los indicadores: imitación, reuniones de trabajo y manual o documentada y, el factor aplicación del conocimiento, que a su vez integra los indicadores: en equipo, rutinaria y documentada.

Respecto al grupo de factores transferencia de conocimiento, estudios recientes muestran que los tres indicadores son importantes para la trasferencia de conocimiento, tal es el caso de Pérez, Leal, Barceló y León (2013), quienes realizan un trabajo cuyo objetivo es el análisis de los procesos de la gestión del conocimiento en las Pymes del sector restaurantero, para una muestra de 12 empresas de México. Los resultados más relevantes sobre estos indicadores son que la imitación es significativa para trasferir el conocimiento, ya que se demostró que el personal está dispuesto a compartir de su conocimiento, todo lo contrario, con el indicador documentada, el cual no es significativo para trasferir el conocimiento.

Algo semejante sucede con Martínez (2013), quien efectuó un trabajo cuyo objetivo es el análisis de la aplicación del conocimiento en las relaciones existentes entre la gestión de conocimiento y la transferencia de conocimiento, para una muestra de 549 empresarios de Venezuela, los resultados más relevantes sobre estos indicadores son que existe una relación significativa entre los indicadores imitación, reuniones de trabajo y capacitación, para trasferir el conocimiento dentro de la organización.

En este sentido, Valencia (2010) realizó una investigación cuyo objetivo fue examinar los principios del modelo de generación y 
transferencia de conocimiento, como son la integridad, aprendizaje, coherencia, participación, flexibilidad y el mejoramiento sistemático para los procesos de dirección y gestión humana, para una muestra de Pymes de la ciudad de Cali, Colombia, los resultados más relevantes sobre estos indicadores son que la capacitación no es relevante para la transferencia del conocimiento, por el contrario los indicadores imitación y reuniones de trabajo los cuales son significativos para trasferir el conocimiento.

Por otra parte, Rodríguez, Pedraja, Delgado, Rodríguez (2010) realizaron un trabajo orientado a identificar relaciones significativas en tres variables: a) la gestión de conocimiento, b) estilos de liderazgo y c) diseño e implementación de la estrategia. Dicho estudio se enfocó a una muestra 78 directivos de Pymes en Chile. En su trabajo sólo integran el indicador trabajo en equipo y sus resultados indican que es significativo para la trasferencia de conocimiento, demostrando que debe existir un liderazgo que impacte positivamente en la trasferencia de conocimiento.

De la misma manera, Valencia (2010), realizó una investigación cuyo objetivo fue examinar los principios del modelo de generación y transferencia de conocimiento, como son la integridad, aprendizaje, coherencia, participación, flexibilidad y el mejoramiento sistemático para los procesos de dirección y gestión humana para una muestra de 20 Pymes en la ciudad de Cali, Colombia, quienes en su trabajo integran solamente la variable trasferencia del conocimiento la cual es significativa para la competitividad de la empresa y motiva a

2 En este tipo de muestreo las muestras no son representativas por el tipo de selección, se dice que son arbitrarias o seleccionadas, donde el investigador formarse en relación con las necesidades reales de la empresa, adquirir nuevos conocimientos, habilidades y crear nuevos procesos para ser más competitivos.

\section{Metodología}

De acuerdo con el portal del Gobierno del Estado de México del año 2017, dicha demarcación está conformada por cinco zonas o macro regiones entre las cuales se encuentran centro, norte, oriente, sur y poniente, de los cuales por sus características geográficas y demográficas se agrupan en 20 regiones socioeconómicas, siendo estas Amecameca, Atlacomulco, Chimalhuacán, Cuautitlán Izcalli, Ecatepec, Huixquilucan, Ixtapan de le Sal, Ixtlahuaca, Lerma, Metepec, Naucalpan, Nezahualcóyotl, Otumba, Tejupilco, Texcoco, Tlalnepantla, Toluca, Tultitlán, Valle de Bravo y Zumpango. Por tanto, la población objeto de estudio que se ha considerado está compuesto por todas las empresas de servicio que residen principalmente en el municipio de Atlacomulco ya que la Zona Norte del Estado de México, está conformada por los siguientes municipios: Acambay, Aculco, El Oro, San Felipe del Progreso, San José del Rincón, Temascalcingo, Timilpán y Atlacomulco.

Cabe destacar que la presente investigación es de carácter cuantitativo, tiene un enfoque descriptivo y utilizó una muestra no probabilística2 direccionada de treinta Pymes del sector servicios ubicadas en un municipio de la Zona Norte del Estado de México. Nos hemos apoyado de la teoría del límite central, la cual de acuerdo con Alvarado y Batanero (2008), tiene gran aplicación en inferencia estadística ya que los

deliberadamente selecciona las unidades representativas de la población, y puede variar de experto a experto (Pimienta, 2000). 
parámetros de las distribuciones de probabilidad pueden expresarse en función de una suma de variables. Así, bajo este planteamiento, se entrevistaron a 30 pequeños y medianos empresarios del sector servicios en el municipio de Atlacomulco, Estado de México.

Se diseñó un instrumento tipo encuesta, elaborado con base en la metodología de Martínez (2013), quedando constituido por 14 ítems con categorías de respuesta en escala de Likert de 1 a 5 puntos, donde el 1 indica totalmente de acuerdo, y el 5 indica totalmente en desacuerdo. Dicho instrumento contiene dos grupos de factores, el primero con indicadores sociodemográficos, y en seguida el grupo de factores de gestión de conocimiento, que a su vez integra dos variables: transferencia $y$ aplicación del conocimiento; ambas con tres indicadores. Cabe precisar que, para efectos del presente trabajo, se muestran los resultados preliminares de la variable transferencia de conocimiento y los tres indicadores que la integran, así como la variable género del grupo de factores sociodemográficos, la tabla 2 muestra las variables de análisis. (Ver Tabla 2)

TABLA 2. VARIABLES DE ANÁLISIS

\begin{tabular}{lll}
\hline Factores & Dimensiones & Indicadores \\
\hline Sociodemográficos & Género & Masculino \\
\cline { 3 - 3 } Gestión de Conocimiento & \multirow{2}{*}{ Transferencia de conocimiento } & Imitación \\
\cline { 2 - 3 } & & Reuniones de trabajo \\
\cline { 2 - 2 } & & Manuales o documentos \\
\hline
\end{tabular}

Fuente: Elaboración propia con base en Martínez (2013).

Es importante mencionar que el proceso de codificación de los tres indicadores queda de la siguiente manera: trasferencia por imitación (CCTI2), el cual mide si el conocimiento que se gestiona se trasfiere por imitación. Así mismo, el indicador transferencia en reuniones de trabajo (CCTRAP1) mide si el conocimiento que se gestiona se trasfiere en reuniones de trabajo en las que se analizan planes. En cuanto al indicador transferencia en reuniones donde se analizan situaciones diversas (CCTRASD6) mide si el conocimiento que se gestiona se trasfiere en reuniones de trabajo en las que se analizan situaciones diversas.

El procedimiento de análisis consistió primeramente en el tratamiento de la

3 Análisis bivariante consiste entre las relaciones de las variables consideradas dos a dos, reconociendo así las distribuciones bidimensionales que se presentan cuando se consideran paralelamente dos caracteres de una misma población o muestra (Sámano, Escamilla \& Velázquez, 2014). información obtenida del estudio de campo, por tanto, se utilizó el programa SPSS Statistics Versión 19 para Ciencias Sociales. Posteriormente se realizó un análisis bivariante3, que consistió en el estadístico tabla de contingencia 4 con la finalidad de detectar la asociación entre las variables aquí consideradas. Dicho estadístico incluye en sus resultados los análisis estadísticos Chicuadrada, los estadísticos nominales $\mathrm{V}$ de Cramer y, correlación de Spearman. Asimismo, se usaron los denominados residuos corregidos, que apoyaron la identificación de las distintas categorías en las variables.

4 Las tablas de contingencia no están estandarizadas, su análisis radica en la interpretación. 


\section{Resultados}

En cuanto al cruce de la variable que corresponde al factor Gestión de Conocimiento, y a la dimensión Transferencia del Conocimiento que integra el indicador Imitación y la dimensión Género, se observa que existe una asociación entre las variables antes mencionadas esto es confirmado por los valores de $x^{2}=(0.049)$ que la hacen significativa, dicho resultado es avalado por la V de Cramer $\rho=(0.04)$. Cabe destacar que los residuos corregidos no son significativos entre los empresarios hombre y mujer, destacando por el valor mostrado en dichos residuos que es (0.2). Sin embargo, se registra una asociación negativa y muy baja entre el indicador imitación y el género $r_{2}=$ $(-0.040)$, tal y como lo muestra la Tabla 3. (Ver Tabla 3).

TABLA 3. CRUCE LA VARIABLE GÉNERO Y EL INDICADOR IMITACIÓN

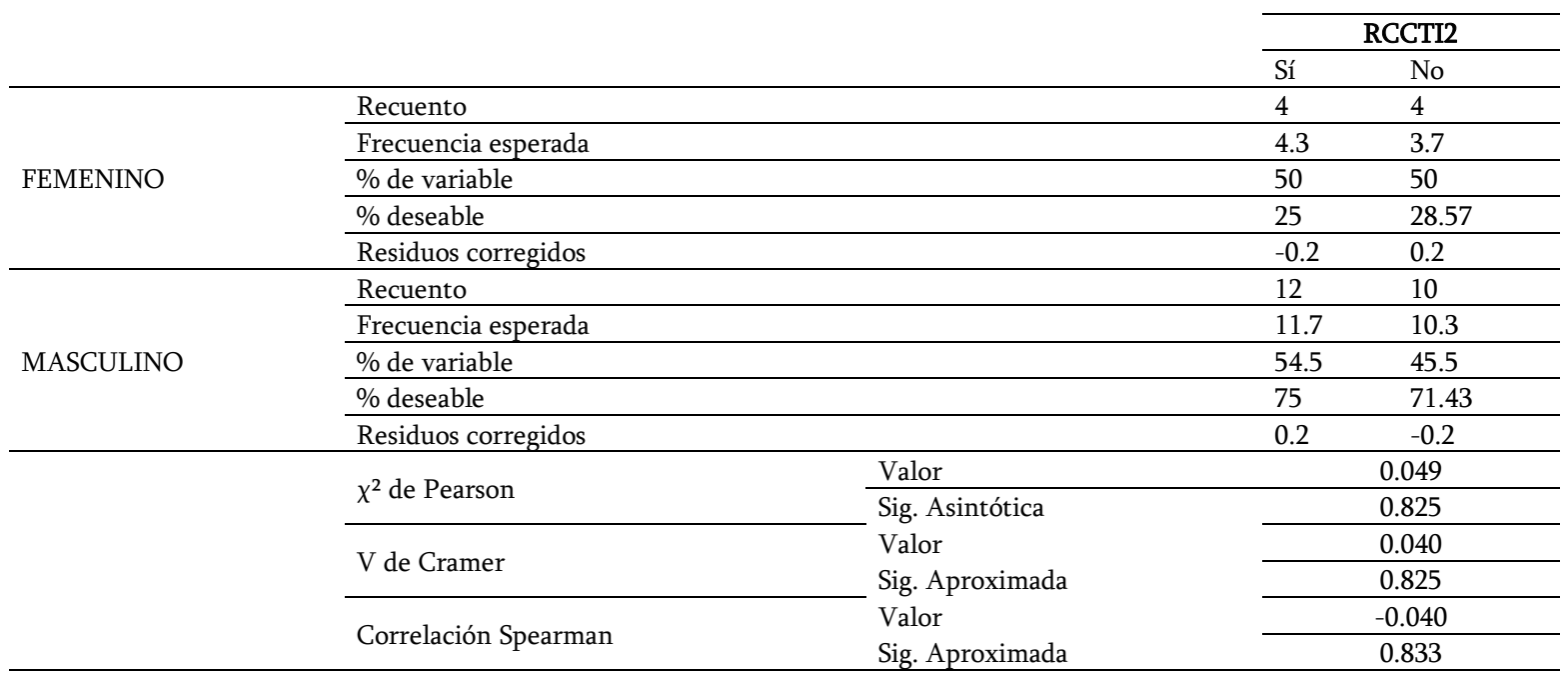

Fuente: Elaboración propia.

En cuanto al cruce de la dimensión Género y el indicador Manual y Documentada que corresponden al factor Gestión del Conocimiento, particularmente a la dimensión Transferencia de Conocimiento, se observa que no es significativa debido a que la $x^{2}=(0.328)$ este resultado es avalado por la V de Cramer el cual corresponde al valor $\rho=(0.111)$ así mismo los residuos corregidos no muestran relación alguna entre las categorías de respuesta que hacen referencia a la obtención de información con base en manuales y documentos. Cabe destacar que los residuos corregidos no son significativos entre los empresarios hombre y mujer, destacando por el valor mostrado por dichos residuos que es (0.6). Sin embargo, se registra una asociación negativa y muy baja entre los indicadores manual y documentada que corresponden al factor Gestión del Conocimiento $r_{2=(-0.111)}$, tal y como se muestra en la Tabla 4. (Ver Tabla 4).

En cuanto al cruce de la dimensión Género y al indicador Reuniones de trabajo que corresponde al factor Gestión del Conocimiento, se observa en la Tabla 5 que no es significativa debido a que su valor es $x^{2}=(1.591)$, el presente resultado es avalado por la $\mathrm{V}$ de Cramer donde su valor es $\rho=(0.230)$. 
Gestión del conocimiento en PYMES del sector servicios: Una aproximación empírica en el Estado de México

TABLA 4. CRUCE DE LA VARIABLE GÉNERO Y EL INDICADOR MANUAL Y DOCUMENTADA

\begin{tabular}{|c|c|c|c|c|}
\hline & & & \multicolumn{2}{|c|}{ RCCTRAP1 } \\
\hline & & & Sí & No \\
\hline \multirow{5}{*}{ FEMENINO } & \multicolumn{2}{|l|}{ Recuento } & 3 & 5 \\
\hline & \multicolumn{2}{|l|}{ Frecuencia esperada } & 3.7 & 4.3 \\
\hline & \multicolumn{2}{|l|}{$\%$ de variable } & 37.5 & 62.5 \\
\hline & \multicolumn{2}{|l|}{$\%$ deseable } & 21 & 31.25 \\
\hline & \multicolumn{2}{|l|}{ Residuos corregidos } & -0.6 & 0.6 \\
\hline \multirow{11}{*}{ MASCULINO } & \multicolumn{2}{|l|}{ Recuento } & 11 & 11 \\
\hline & \multicolumn{2}{|l|}{ Frecuencia esperada } & 10.3 & 11.7 \\
\hline & \multicolumn{2}{|l|}{$\%$ de variable } & 50 & 50 \\
\hline & \multicolumn{2}{|l|}{$\%$ deseable } & 79 & 68.75 \\
\hline & \multicolumn{2}{|l|}{ Residuos corregidos } & 0.6 & -0.6 \\
\hline & \multirow{2}{*}{$\chi^{2}$ de Pearson } & Valor & 0.368 & \\
\hline & & Sig. Asintótica & 0.544 & \\
\hline & \multirow{2}{*}{ V de Cramer } & Valor & 0.111 & \\
\hline & & Sig. Aproximada & 0.544 & \\
\hline & \multirow{2}{*}{ Correlación Spearman } & Valor & -0.111 & \\
\hline & & Sig. Aproximada & 0.560 & \\
\hline
\end{tabular}

Fuente: Elaboración propia.

Por lo tanto, los residuos corregidos no dan a conocer que el conocimiento se transfiere a través de situaciones diversas que se van dando en las empresas, ya que se obtiene el $(-1.3)$. Sin embargo, se registra una asociación baja y muy baja entre la dimensión Género y al indicador Reuniones de trabajo que corresponde al factor Gestión del Conocimiento negativa $r_{2=(-0.230)}$, tal y como lo muestra la Tabla 5. (Ver Tabla 5).

TABLA 5. CRUCE DE LA VARIABLE GÉNERO Y REUNIONES DE TRABAJO

\begin{tabular}{|c|c|c|c|c|}
\hline & & & \multicolumn{2}{|c|}{ RCCTRASD6 } \\
\hline & & & Sí & No \\
\hline \multirow{5}{*}{ FEMENINO } & \multicolumn{2}{|l|}{ Recuento } & 1 & 7 \\
\hline & \multicolumn{2}{|l|}{ Frecuencia esperada } & 2.4 & 5.6 \\
\hline & \multicolumn{2}{|l|}{ \% de variable } & 12.5 & 87.5 \\
\hline & \multicolumn{2}{|l|}{$\%$ deseable } & 11 & 33.33 \\
\hline & \multicolumn{2}{|l|}{ Residuos corregidos } & -1.3 & 1.3 \\
\hline \multirow{5}{*}{ MASCULINO } & \multicolumn{2}{|l|}{ Recuento } & 8 & 14 \\
\hline & \multicolumn{2}{|l|}{$\begin{array}{l}\text { Frecuencia esperada } \\
\end{array}$} & 6.6 & 15.4 \\
\hline & \multicolumn{2}{|l|}{$\%$ de variable } & 36.4 & 63.6 \\
\hline & \multicolumn{2}{|l|}{$\%$ deseable } & 89 & 66.67 \\
\hline & \multicolumn{2}{|l|}{ Residuos corregidos } & 1.3 & -1.3 \\
\hline & \multirow{2}{*}{$\chi^{2}$ de Pearson } & Valor & 1.591 & \\
\hline & & Sig. Asintótica & 0.207 & \\
\hline & \multirow{2}{*}{ V de Cramer } & Valor & 0.230 & \\
\hline & & Sig. Aproximada & 0.207 & \\
\hline & \multirow{2}{*}{ Correlación Spearman } & Valor & -0.230 & \\
\hline & & Sig. Aproximada & 0.221 & \\
\hline
\end{tabular}

Fuente: Elaboración propia.

\section{CONCLUSIONES}

A partir del objetivo planteado en el presente trabajo, se concluye que, en cuanto al grupo de indicadores (Imitación,
Reuniones de trabajo y Manuales o documentos) del factor Transferencia de Conocimiento, la Imitación es significativa para el grupo de empresarios mujeres y hombres de esta muestra, tal y como señalan 
estudios anteriores como el de Pérez, Leal, Barceló y León (2013), Martínez (2013) y Valencia (2010), quienes indican que dicha variable es estadísticamente significativa, por tanto este resultado encontrado en el estudio, pueda deberse a que el sistema de aprendizaje a través de la imitación sea más común y se realiza de manera empírica dentro de las Pymes.

Gutiérrez et al. (2015) en su investigación del sector de servicios a 30 Pymes de la ciudad de Chihuahua, resaltan las dimensiones de socialización, interiorización, exteriorización y combinación para la generación y conversión del conocimiento tácito a explícito; en donde su variable socialización y exteriorización dio como resultado imitación al momento de tener la siguiente afirmación -Se permite y se alienta el diseño de procesos resultado de la observación de la competencia- se interpretó -No visitamos a la competencia, pero Sí los observamos para copiarles-. En la dimensión de exteriorización afirmaron: -Se permite la imitación de procesos resultado de la observación de la competencia- con estos resultados se concluye que la variable Imitación es la forma de generar y transferir el conocimiento de forma tácita.

Por otra parte, la variable Reunión de trabajo se identifica que no es significativa para el grupo de empresarios mujeres $y$ hombres de esta muestra, sin embargo, en el análisis bivariante, se visualiza que para los varones es más significativo que para las mujeres, esto quizás pueda beberse a que en su mayoría los empresarios son hombres representando un 73 por ciento de la muestra.

Estas premisas permiten constatar la teoría de creación del conocimiento organizacional en donde adoptan la definición tradicional como creencia cierta justificada (Montreal, 2000) es decir que lo que observan, experimentan y analizan los individuos, lo interiorizan y lo llevan a la práctica diaria, dando como resultado un modelo mental que influye en el comportamiento y la acción.

La transmisión del conocimiento forma parte de la vida natural de una organización, ya que se orienta en capturar y sacar provecho de la experiencia colectiva para ser accesible a cualquier miembro de esta. Evidentemente este proceso puede ser positivo, cuando una persona comparte su patrimonio (conocimiento) de manera voluntaria, en aras de fortalecer a la organización; y por el otro lado tenemos la parte negativa, cuando las personas no desean ni permiten la compartición de los saberes a terceros, ya que suelen tener resistencia al cambio derivado de factores como el miedo, la inseguridad, desconfianza en sí mismos, lo que ocasiona que el conocimiento se continúe dando de forma empírica y en ocasiones se pierda o se vuelva obsoleto.

Otro factor que impide la transferencia de conocimiento es que las personas tienen sobre carga de información, pero no de conocimiento, por tal motivo la gestión de conocimiento puede convertirse en una vía real para dar sentido a toda esa información que llega a un puesto de trabajo (Vargas \& Moreno, 2005). Por lo anterior, se propone la creación de articulaciones estratégicas para dotar de sentido a la información, volviéndola conocimiento útil y accesible, dónde $\mathrm{y}$ cuándo se necesite mediante justamente las actividades de gestión y transferencia del conocimiento.

De acuerdo con Ordoñez (2001), las empresas en general no adoptan un enfoque uniforme para la gestión del conocimiento, 
no obstante, se enfocan en establecer estrategias para su generación y transferencia en diversos niveles organizacionales, en la búsqueda de la creación de una ventaja competitiva sostenible.

De esta manera, es recomendable la identificación de las diversas etapas de socialización, exteriorización, combinación e internalización del conocimiento, las cuales se asocian al saber qué (know-what), relacionado con los hechos; saber por qué (know-why) conocimiento científico por excelencia como mente humana-sociedad; saber cómo (know-how) habilidad y/o capacidad para hacer algo; saber quién (know-who) diferentes habilidades, obteniendo un modelo holístico de conocimiento es decir el ADN de las organizaciones a partir de la implementación de estrategias de gestión del conocimiento (Gutiérrez et al. 2015; Angulo, 2016 y Pérez, 2016).

Dichas estrategias deben ir ligadas a un proceso que favorezca la gestión del conocimiento, deben también profundizar en el proceso de socialización, lo cual permita intercambiar y compartir conocimiento, lo cual requiere la interiorización, es decir, dotarlo de significado, y que el individuo lo aprehenda y lo haga suyo, muchas veces a través del trabajo en equipo o bien del dominio personal (García, 2016; Montreal, 2000).

Siguiendo este orden de ideas, como lo indican Maldonado, Martínez y García (2012), para que las Pymes sean competitivas tendrán que implementar estrategias empresariales tendientes a gestionar adecuadamente tanto el conocimiento tácito como el implícito, para lo cual es preciso incentivar la compartición de conocimientos, habilidades y experiencias entre los colaboradores, además de dotar a la organización de políticas que reconozcan esta actividad.

En la actualidad, las organizaciones inteligentes son aquellas preocupadas en la creación, gestión y transferencia de conocimiento a manera de un nuevo paradigma centrado en las competencias, capacidades, y habilidades de las personas como recursos estratégicos con los cuales sustentan la consecución de una ventaja competitiva sostenible.

Esta investigación nos permitió identificar los indicadores más significativos del factor gestión del conocimiento en Pymes del sector servicios en un municipio de la Zona Norte del Estado de México, encontrando que la imitación resulta el indicador más significativo para los pequeños y medianos empresarios de la muestra de la investigación, lo cual alerta acerca de la falta de planeación para el tema de gestión de conocimiento en este tipo de empresas.

Por lo anterior, se considera esta una relevante aportación para comprender cómo se da la gestión del conocimiento en las Pymes en una región específica de México. Una de las principales limitaciones para el desarrollo fue justamente que no se incluyeron los catorce indicadores considerados en el modelo de Martínez (2013) sino solo tres, así mismo, otra limitación fue la escasa literatura en la región acerca de este tema, por lo cual como futuras líneas de investigación se espera realizar un profundo análisis bibliométrico a fin de detectar otros indicadores relevantes en este tema, para el desarrollo de una metodología adaptada al contexto latinoamericano, el cual 


\section{Caldera-González, Diana del Consuelo, Escamilla-Salazar, Zugaide y Ruiz-Lara, Fortino}

pueda ser aplicado y validado con una muestra más grande.

\section{REFERENCIAS}

Alvarado, H. \& Batanero, C. (2008). Significado del teorema central del limite en textos universitarios de probabilidad y estadística. Estudios pedagógicos (Valdivia), 34(2), 7-28. doi: 10.4067/S0718-07052008000200001

Angulo, R. (2016). Gestión del conocimiento y aprendizaje organizacional: una visión integral. Informes psicológicos, 17(1), 53-70. doi: http://dx.doi.org/10.18566/infpsic.v17n1a03

Arriaga, R., \& Gómez, M. D. (2014). Estrategias de gestión del conocimiento para generar ventajas competitivas en pequeñas y medianas empresas en el Estado de México. Gestión y estrategia (46), 55-72.

Bagnoli, C., \& Vedovato, M. (2012). The impact of knowledge management and strategy configuration coherence on SME performance. Journal of Management \& Governance,18(2),615-647.

Banco del Ahorro Nacional y Servicios Financieros, S.N.C. [BANSEFI] (27 de junio de 2018). Día de las microempresas y las pequeñas y medianas empresas. Obtenido de https://www.gob.mx/bansefi/articulos/diade-las-microempresas-y-las-pequenas-y-medianasempresas?idiom $=\mathrm{es}$

Barrios, K., Olivero, E., \& Acosta, J. C. (2017). Gestión del conocimiento y capacidad de innovación. Modelos, Sistemas y Aplicaciones. Ediciones Universidad Simón Bolívar. Barranquilla: Universidad Simón Bolívar.

Bernal, T., Fracica, N. \& Frost. G. (2012). Análisis de la relación entre la innovación y la gestión del conocimiento con la competitividad empresarial en una muestra de empresas en la ciudad de Bogotá. Estudios Gerenciales, 28(1), 303315.

Bustelo, C., \& Amarilla, R. (2001). Gestión del conocimiento y gestión de la información, Inforarea S.L., Boletín del Instituto Andaluz de Patrimonio Histórico, 226-230.

Byung H.S., Hyunsoo, L., \& Tae, S. K. (2018). A study on knowledge management system for knowledge competitiveness with one stop knowledge service. 1st IEEE International Conference on Knowledge Innovation and Invention (ICKII). doi: 10.1109/ICKII.2018.8569149

Cabrera, A., \& Rincón, M. (2001). La gestión del conocimiento: creando competitividad en la nueva economía. ICE Nueva Economía y Empresa, 79-91.

Cabrera, R., Medina, A., Abab, J., Nogueira, D., \& Núñez, Q. (2015). La integración de sistemas de gestión empresariales, conceptos, enfoques y tendencias. Sistema de información Científica, 46(3), 3-8.
Črnjar, K. (2006). Contribution of knowledge management to the development of the hotel enterprise's competitiveness. Societal studies, 6(3), 557-578.

Cuevas, H., Rangel, J. \& Hernández, O. (2014). La influencia de las actividades de innovación y la gestión del conocimiento en la competitividad de las Pymes manufactureras un estudio empírico. En Ramos M. \& Solís M (ed.), Desarrollo económico en el crecimiento Empresarial, (pp. 53-68). Bolivia: ECORFAN.

Dalkir, K. (2005). Knowledge management in the theory and practice. Oxford: Elsevier.

Davidson, C., \& Voss, P. (2002). Knowledge management: an introduction to creating competitive advantage from intellectual capital. Auckland, NZ: Tandem Press.

Escamilla, S.Z., Rodríguez, A.L., \& Gómez, M. (2010). Factores que se relacionan con la intención empresarial: Una perspectiva de género. Comunicación presentada en el $V$ Internacional Workshop of Research based on GEM. Cadiz, Junio.

García, F. J. (2016). La socialización como proceso clave en la gestión del conocimiento. Education in the Knowledge Society, 17(2), 7-14

Gutiérrez, M. D., Sapién, A. L., \& Piñón, L. C. (2015). Gestión del conocimiento en PyME del sector servicios en la ciudad de Chihuahua. Nova Scientia, 15(7), 499-513.

Heredia, L., García, D., \& Bastida, F. (2008). Los sistemas de control de gestión y la innovación: Su efecto sobre el rendimiento de las Pymes. Actualidad Contable Faces, 11(17) 135-152.

Hernández, R., Fernández, C., \& Baptista, P. (2006). Metodología de la investigación. México: McGraw Hill.

Instituto Nacional de Estadística y Geografía [INEGI]. (2016). Encuesta nacional sobre productividad y competitividad de las micro, pequeñas y medianas empresas (ENAPROCE) 2015. Recuperado de https://www.inegi.org.mx/contenidos/programas/enaproc e/2015/doc/ENAPROCE_15.pdf

Jingjing, L. (2007). Sharing knowledge and creating knowledge in organizations: the modeling, implementation, discussion and recommendations of weblog-based knowledge management. International Conference on Service Systems and Service Management, Chengdu, 2007, pp. 1-6. doi: 10.1109/ICSSSM.2007.4280206

López-Inda, Karina Azucena, Alvarado Altamirano, Sergio, Fong Reinoso, Carlos \& González Álvarez, Eleazar (2019). Capital social en la perspectiva de la teoría de recursos y capacidades: un análisis bibliométrico. Inquietud Empresarial, 19(1), 63-77.

Maldonado, G., Martínez, M. D., \& García, R. (2012). Gestión del Conocimiento y crecimiento en la Pyme manufacturera de Aguascalientes (México). Cuadernos de Administración, 28(47), 25-36. 


\section{Gestión del conocimiento en PYMES del sector servicios: Una aproximación empírica en el Estado de México}

Martínez, M. (2013). Desarrollo de un modelo de gestión del conocimiento en la cadena de suministro de la industria agroalimentaria. Tesis Doctoral, E.T.S.I. Industriales (UPM).

Marulanda, C.E., Giraldo, J.A., \& López, M. (2012). Herramienta para medición de la gestión del conocimiento en las Pymes de Colombia. Revista virtual Católica del Norte, (35), 83-104.

Marulanda, C. E., López, M., \& Mejía, M. (2013). Análisis de la gestión del conocimiento en Pymes de Colombia. Gerencia Tecnológica Informática, 12(33), 33-43.

Mirabal, J. (2015). Gestión dinámica de conocimiento organizacional. Revista Venezolana de Información, Tecnología y Conocimiento, 12(2), 55-78.

Montoya, A. (2013). Análisis de la importancia del capital humano y social en las expectativas de crecimiento en empresarias de algunos municipios de la zona norte del estado de México. Tesis de licenciatura no publicada, UAEM, Atlacomulco.

Montreal, J. (2000). Gestión del conocimiento y capital intelectual. Centro de Investigación y Documentación sobre problemas de la Economía, el Empleo y las Cualificaciones. Donostia San Sebastián: España.

Nagles, G. N. (2007). La gestión del conocimiento como fuente de innovación. Revista Escuela de Administración de Negocios, (61), 97-102.

Organización de Naciones Unidas [ONU]. (2018). Día de las Microempresas y las Pequeñas y Medianas Empresas, 27 de junio. Obtenido de http://www.un.org/es/events/smallbusinessday/backgrou nd.shtml

Ordoñez, P. (2001). La gestión del conocimiento como la base para el logro de una ventaja competitiva sostenible: la organización occidental versus japonesa. Investigaciones Europeas de Dirección y Economía de la Empresa, 7(3), 91-108.

Pérez, A., Leal, V, Barceló, M., \& León, J. (2013). Un diagnóstico de la gestión del conocimiento en las Pymes del sector restaurantero para identificar áreas de mejora en sus procesos productivos. Intangible capital, 9(1), 153-183.

Pérez, M. (2009). Gestión del conocimiento en las organizaciones: fundamentos, metodología y praxis. El profesional de la información, 18(1), 111-113.

Pimienta, L. R. (2000). Encuestas probabilísticas vs. no probabilísticas. Política y Cultura, 13, 263-276.

Rodríguez, D. (2006). Modelos para la creación y gestión del conocimineto: una aproximación teórica. Educar, 25-39.

Rodríguez, E., Pedraja, L., Delgado, M. \& Ponce, J. (2010). Gestión del conocimiento, liderazgo, diseño e implementación de la estrategia: un estudio empírico en pequeñas y medianas empresas. Revista chilena de ingeniería, 18(3), 373-382.

Ruiz, J., \& García, M. (2013). Gestión del conocimiento e innovación. España: INAP.

Sámano, Á., Escamilla, Z., \& Velázquez, E. (2014). Prácticas de responsabilidad social en materia ambiental en MiPymes florícolas y financiamiento: una aproximación empírica. Investigación administrativa, 43(114), 67-81.

Valencia, M. (2010). Modelo de generación y transferencia de conocimiento para los procesos de dirección y gestión humana en Pymes del sector cárnicos de la ciudad de Cali. Ingeniería Industrial, 30(3), 1-6.

Vargas, A., \& Moreno, M.J. (2005). La gestión del conocimiento en las organizaciones. Tourisim \& Management Studies, 1, 139-151.

Wanling, F., Jiasu, L., y Jinyu, W. (2001). Business management with knowledge management as the core under knowledge economy. PICMET '01. Portland International Conference on Management of Engineering and Technology. Proceedings 1: Book of Summaries (IEEE Cat. No.01CH37199). doi: 10.1109/PICMET.2001.951762

Wilson, T. (2002). The nonsense of knowledge management. Information Research, 8 (1), 144-154.

Yonghai, Z., Aiqun, Z., Houlei, T., y Shugang, C. (2009). On enterprise knowledge management practice based on organizational learning. Proceedings of the 2009 Second International Symposium on Knowledge Acquisition and Modeling, 02(316-318). doi.org/10.1109/KAM.2009.183 